\title{
FGF19 action in the brain induces insulin-independent glucose lowering
}

\author{
Gregory J. Morton, ${ }^{1}$ Miles E. Matsen, ${ }^{1}$ Deanna P. Bracy, ${ }^{2}$ Thomas H. Meek, ${ }^{1}$ Hong T. Nguyen, ${ }^{1}$ \\ Darko Stefanovski, ${ }^{3}$ Richard N. Bergman, ${ }^{3}$ David H. Wasserman, ${ }^{2}$ and Michael W. Schwartz ${ }^{1}$ \\ 1Diabetes and Obesity Center of Excellence, Department of Medicine, University of Washington, Seattle, Washington, USA \\ 2Department of Molecular Physiology and Biophysics, Vanderbilt School of Medicine, Nashville, Tennessee, USA. \\ ${ }^{3}$ Diabetes and Obesity Research Institute, Cedars-Sinai Medical Center, Los Angeles, California, USA.
}

\begin{abstract}
Insulin-independent glucose disposal (referred to as glucose effectiveness [GE]) is crucial for glucose homeostasis and, until recently, was thought to be invariable. However, GE is reduced in type 2 diabetes and markedly decreased in leptin-deficient $o b / o b$ mice. Strategies aimed at increasing GE should therefore be capable of improving glucose tolerance in these animals. The gut-derived hormone FGF19 has previously been shown to exert potent antidiabetic effects in $o b / o b$ mice. In $o b / o b$ mice, we found that systemic FGF19 administration improved glucose tolerance through its action in the brain and that a single, low-dose i.c.v. injection of FGF19 dramatically improved glucose intolerance within 2 hours. Minimal model analysis of glucose and insulin data obtained during a frequently sampled i.v. glucose tolerance test showed that the antidiabetic effect of i.c.v. FGF19 was solely due to increased GE and not to changes of either insulin secretion or insulin sensitivity. The mechanism underlying this effect appears to involve increased metabolism of glucose to lactate. Together, these findings implicate the brain in the antidiabetic action of systemic FGF19 and establish the brain's capacity to rapidly, potently, and selectively increase insulin-independent glucose disposal.
\end{abstract}

\section{Introduction}

In addition to insulin secretion and insulin sensitivity (Si), insulin-independent mechanisms are critical to normal glucose homeostasis (1). Given that such mechanisms contribute at least as much to normal glucose tolerance as does insulin itself (1), it is surprising how little is known about them. This lack of research interest can be traced to the widespread perception of insulin-independent glucose disposal, which has been termed glucose effectiveness (GE; the ability of glucose to promote its own disposal, independently of insulin), as the fixed, obligate, and unregulated mechanism whereby insulin-insensitive tissues meet ongoing fuel needs. Yet glucose intolerance and type 2 diabetes (T2D) are characterized by decreases of GE as well as of insulin secretion and action (1). In leptin-deficient $o b / o b$ mice, for example, GE was shown recently to be reduced by approximately $70 \%$, based on minimal model analysis of data obtained from a frequently sampled i.v. glucose tolerance test (FSIGT) (2). Based on its importance to glucose homeostasis, we hypothesized that any intervention capable of normalizing glucose tolerance in these mice should do so, at least in part, by increasing GE.

FGF19 and its rodent homolog, FGF15, is one of three members of the family of hormonal FGFs. FGF19 is secreted by enterocytes located in the distal small intestine following activation of the nuclear bile acid receptor, FXR, by bile acid binding (3). In addition to its well-established role in the negative feedback control of hepatic bile acid synthesis (4, 5), FGF19 exerts potent antidiabetic effects in rodent models, including $o b / o b$ mice (6). Similarly, transgenic overexpression of FGF19 improves glucose tolerance in diet-induced obese mice $(6,7)$, whereas FGF15-deficient mice display impaired glucose tolerance that is corrected by FGF19 administration (8).

Conflict of interest: The authors have declared that no conflict of interest exists. Citation for this article: JClin Invest. 2013;123(11):4799-4808. doi:10.1172/JCI70710.
Although initially thought to act primarily via FGFR4 receptors in the liver, the antidiabetic effects of FGF19 appear to involve a different FGF receptor subtype, because they are preserved in FGFR4-deficient mice (9). Instead, several findings implicate FGFR1 in this effect. First, systemic administration of a FGF19 variant that activates FGFR1 but not FGFR4 ameliorates diabetes in $o b / o b$ mice, while this effect is absent for a FGF19 variant that activates FGFR4 but not FGFR1 (10). Second, the potent glucose-lowering effect of FGF19 is mimicked by monoclonal antibodies that selectively activate a specific FGFR1 isoform (FGFR1c) (11). Although activation of FGFR1 in peripheral tissues, such as brown and white adipose tissue, may contribute to glucose lowering, this receptor is also expressed in mediobasal hypothalamic areas involved in glucose homeostasis $(12,13)$, and administration of FGF19 directly into the brain improves glucose tolerance in both $o b / o b$ mice (6) and diet-induced obese rats (14).

The current studies were undertaken to determine whether the antidiabetic effect of systemically administered FGF19 involves a central site of action and, if so, to determine the contribution(s) made by insulin-dependent and -independent mechanisms to this effect. We report that, in $o b / o b$ mice, the glucose-lowering effect of systemic FGF19 is reduced by approximately $50 \%$ when its action in the brain is blocked by i.c.v. administration of an FGFR antagonist. Thus, the brain plays a key role in the antidiabetic effects of this hormone. To investigate how FGF19 action in the brain affects systemic glucose metabolism, we performed a FSIGT 2 hours after i.c.v. injection of either FGF19 or its vehicle in $o b / o b$ mice as well as in vehicle-injected C57BL/ 6 controls. Glucose and insulin data from the FSIGT were analyzed using the minimal model method, which has been widely used in humans (1) and was recently validated in mice (including in $o b / o b$ mice) (2), as a tool with which to measure insulin secretion, Si, and GE. Surprisingly, we found that the potent antidiabetic effect of i.c.v. FGF19 in $o b / o b$ mice did not involve increases of either insulin secretion or Si. Instead, the 

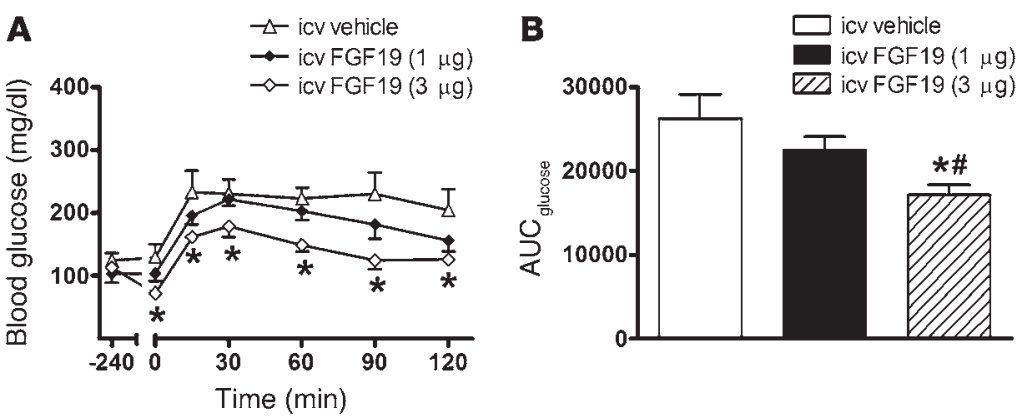

\section{Figure 1}

Effect on glucose tolerance of a single injection FGF19 given either centrally or systemically to $o b / o b$ mice. (A and C) Glucose tolerance $(0.5 \mathrm{~g} / \mathrm{kg})$ and (B and D) the integrated area under the glucose curve $\left(\mathrm{AUC}_{\text {glucose }}\right)$ in leptin-deficient $o b / o b$ animals that received either a single, acute i.c.v. or i.p. injection of FGF19 at either 1 or $3 \mu \mathrm{g}$ or its vehicle. Data represent mean \pm SEM. ${ }^{*} P<0.05$ vs. i.c.v. vehicle. ${ }^{\#} P<0.05$ vs. i.c.v. FGF19 $(1 \mu \mathrm{g})$.
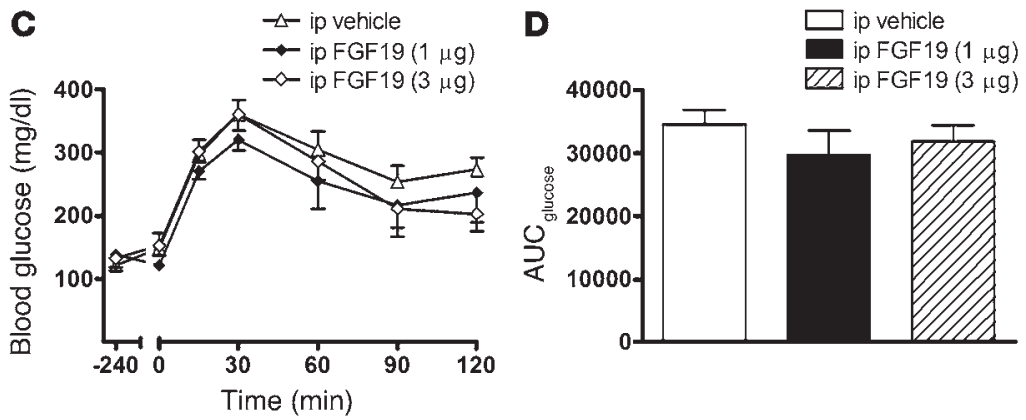

effect was mediated entirely by a 3-fold increase of GE. Furthermore, our data suggest that reduced GE in $o b / o b$ mice is linked to reduced glycolysis of glucose to lactate and that FGF19 action in the CNS of these animals reverses this defect. Thus, whereas insulin-independent glucose lowering has heretofore been considered invariant, obligate, and unregulated, our findings reveal the brain's inherent capacity to rapidly, potently, and selectively increase glucose disposal via this mechanism.

\section{Results}

Effect of i.c.v. FGF19 on glucose tolerance in ob/ob mice. Since i.c.v. FGF19 can reduce food intake and body weight as well as blood glucose levels $(6,14)$, we initially sought to identify a protocol in which effects on glucose metabolism are not confounded by changes of energy balance. After verifying an earlier report (6) showing that chronic (i.e., over 6 days) i.c.v. administration of FGF19 exerts potent glucose lowering in $o b / o b$ mice (data not shown), we asked whether this effect can be detected following just a single i.c.v. injection of FGF19, with food removed during the interval (1.5 hours) between i.c.v. injection and glucose tolerance testing. We found that, in $o b / o b$ mice (mean body weight: $49.8 \pm$ $1.2 \mathrm{~g}$ ), i.c.v. injection of FGF19 lowered fasting blood glucose levels $(P<0.05)$ and dose-dependently improved glucose tolerance relative to that of vehicle-treated controls $(P<0.05)$ (Figure 1, A and B). Since the mice did not have access to food following i.c.v. injection, the observed effects were not due to changes of food intake or body composition, nor were they due to leakage of FGF19 into the periphery, since systemic administration at the same low dose had no effect on these parameters (Figure 1, C and D). Thus, FGF19 action in the brain induces glucose lowering via a mechanism that is unrelated to changes of energy balance.

Role of the brain in the response to systemic FGF19. Most studies of the antidiabetic effect of FGF19 have focused on effects in peripheral tissues. To determine whether the brain also plays a role, we asked whether the response to systemic FGF19 is attenuated by central administration of an FGF receptor inhibitor (PD173074).
Prior to undertaking this study, we verified that, when given i.c.v., the inhibitor blocks the response to i.c.v. FGF19. Consistent with our earlier observations, a single i.c.v. injection of FGF19 lowered fasting blood glucose levels and significantly improved i.p. glucose tolerance in $o b / o b$ mice that received an i.c.v. vehicle pretreatment injection, and, as expected, this beneficial effect was fully blocked by i.c.v. pretreatment with PD173074 at a dose that had no effect when given alone (Figure 2, A and B).

We next asked whether i.c.v. pretreatment with the FGFR inhibitor affects glucose lowering induced by systemic administration of FGF19 at a dose far higher than what is needed when given i.c.v. $(1 \mathrm{mg} / \mathrm{kg} v \mathrm{~s} .3 \mu \mathrm{g})$. As expected, systemic administration of FGF19 lowered fasting blood glucose levels and improved glucose tolerance in $o b / o b$ mice relative to that of vehicle-treated controls. Since this effect was attenuated by approximately $50 \%$ by i.c.v. pretreatment with PD173074 (Figure 2, C and D), the glucose-lowering effect of systemic FGF19 appears to be mediated at least in part via a central mechanism.

Effect of i.c.v. FGF19 on determinants of i.v. glucose tolerance in $o b / o b$ mice. Having established a role for the brain in the antidiabetic effect of FGF19, we next sought to investigate underlying mechanisms. To this end, we used the FSIGT protocol that, having been used in human studies for many years (1), was recently validated for use in mice, including $o b / o b$ mice (2). Analysis of glucose and insulin data from the FSIGT using the minimal model provides quantitative estimates of insulin secretion, GE, Si, and the disposition index (a measure of the ability of $\beta$ cells to compensate for insulin resistance). Three groups of mice were studied: $o b / o b$ mice treated with a single i.c.v. injection FGF19 ( $3 \mu \mathrm{g}$ ) or its vehicle (mean body weight: $44.2 \pm 0.8 \mathrm{~g}$ ) and WT C57BL/ 6 control mice that received i.c.v. vehicle (weight: $25.1 \pm 0.5 \mathrm{~g}$ ). This approach allowed us to determine (a) whether the glucoregulatory effects of central FGF19 in $o b / o b$ mice are due to increases of insulin secretion, insulin action, or insulin-independent glucose disposal and (b) the extent to which glucose tolerance of $o b / o b$ mice was normalized following a single i.c.v. injection of FGF19. While fasting blood glucose 
A
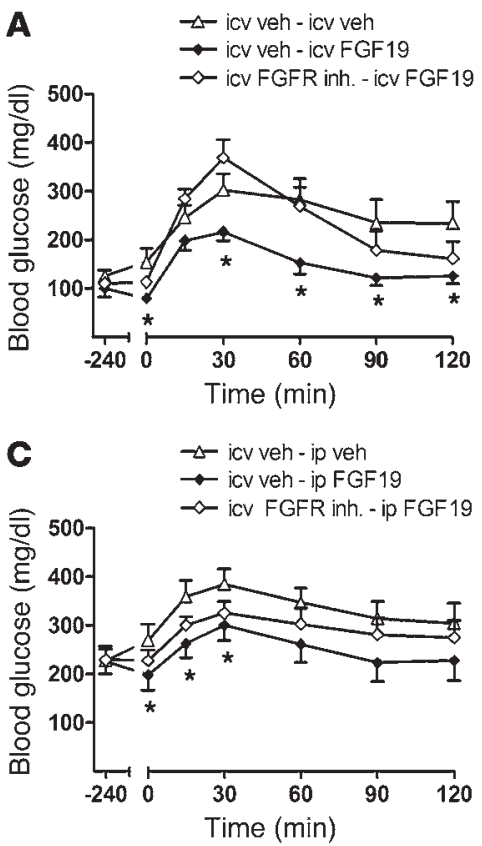

B

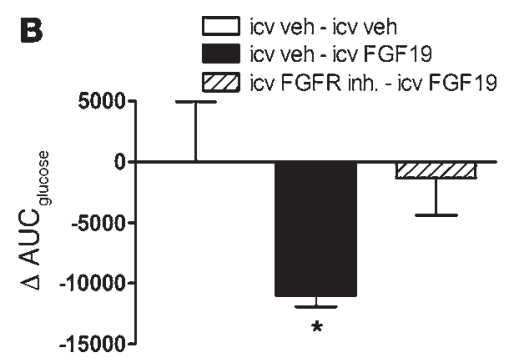

\section{Figure 2}

Effect of a centrally administered FGF receptor blocker on the glucose-lowering effect of FGF19. (A and C) Glucose tolerance $(0.5 \mathrm{~g} / \mathrm{kg})$ and $(\mathbf{B}$ and $\mathbf{D})$ the change in the integrated area under the glucose curve in leptin-deficient $o b / o b$ mice that received either an i.c.v. pretreatment injection of the FGFR inhibitor, PD173074 $(25 \mu \mathrm{g})$, or its vehicle (veh) 1-hour prior to either systemic or central administration of FGF19 or its vehicle. Animals were then subject to an ipgtt $(0.5 \mathrm{~g} / \mathrm{kg}) 90$ minutes later. Data represent mean \pm SEM. ${ }^{\star} P<0.05$ vs. i.c.v. vehicle-i.c.v./i.p. vehicle.

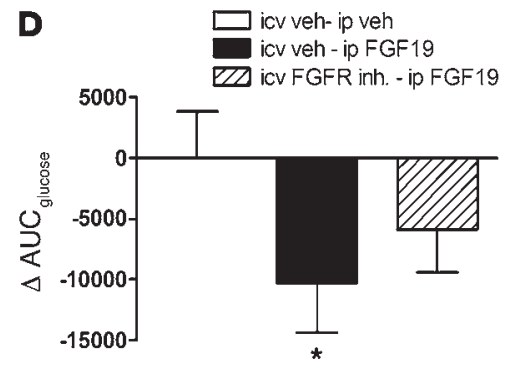

levels were similar in i.c.v. vehicle-treated $o b / o b$ and WT mice, basal plasma insulin levels were markedly elevated in the former group, consistent with previous evidence of severe insulin resistance in these animals (2). Similarly, glucose tolerance was markedly impaired in i.c.v. vehicle-treated $o b / o b$ mice relative to that in WT controls following an i.v. glucose bolus (Figure 3, A and B), despite a markedly increased acute insulin response to glucose (a measure of first-phase insulin secretion) and sustained elevation of plasma insulin levels throughout the study (Figure 3, C and D).

As expected, this marked impairment of glucose tolerance was significantly improved in $o b / o b$ mice that received FGF19 $(P<0.05)$ (Figure 3, A and B). Based on the area under the glucose curve, i.c.v. FGF19 treatment corrected the abnormality of glucose tolerance of $o b / o b$ mice by $65 \%(P<0.05)$. Therefore, within 2 hours of a single i.c.v. injection FGF19 dramatically improved glucose tolerance in $o b / o b$ mice. As this improvement occurred despite no difference in plasma insulin levels at any time during the glucose tolerance test (Figure 3, C and D), it cannot be attributed to increased insulin secretion. We therefore turned our attention to its effects on the two other determinants of glucose tolerance, $\mathrm{Si}$ and insulin-independent glucose disposal. As expected (2), minimal model analysis of glucose and insulin data from the FSIGT revealed the Si index to be markedly reduced in i.c.v. vehicle-treated $o b / o b$ mice relative to that in WT controls (Figure 3E). Unexpectedly, however, i.c.v. FGF19 injection had no effect on Si in $o b / o b$ mice. Thus, the observed improvement of glucose tolerance mediated by i.c.v. FGF19 was not due to increased Si (Figure 3E), and since insulin secretion was similarly unaffected, the improvement was also not associated with an increase of the disposition index (data not shown).

As previously reported (2), GE (defined as the ability of an increased concentration of glucose to promote its own disposal at basal insulin) was reduced by approximately $80 \%$ in i.c.v. vehicle-treated $o b / o b$ mice relative to that in WT mice (Figure $3 \mathrm{~F}$ ). Given the importance of GE as a determinant of glucose tolerance (1), this decrease undoubtedly plays a major role in the impaired glucose homeostasis of $o b / o b$ mice. This assertion in turn predicts that an increase of GE has the potential to explain the beneficial effect of central FGF19. Consistent with this prediction, we found that i.c.v. FGF19 induced a 3-fold increase of GE, such that it was almost completely normalized (Figure 3F). Thus, the effect of i.c.v. FGF19 to improve glucose tolerance in $o b / o b$ mice results primarily from a marked increase of GE. Under the influence of FGF19, therefore, the brain has the capacity to rapidly, potently, and selectively increase insulin-independent glucose disposal.

As a first step to better understand how the CNS action of FGF19 action increases GE, we measured plasma lactate levels during the FSIGT, as described in Figure 3. Previous work has shown that, in response to a glucose challenge, plasma lactate levels increase as a consequence of glucose uptake into tissues (primarily liver), followed by glycolysis to lactate that is subsequently released into the circulation. Since both GE and the plasma lactate response to glucose are reduced in obese individuals (15-17), we considered the possibility that, in $o b / o b$ mice, reduced GE is causally linked to decreased metabolism of injected glucose to lactate. Consistent with this hypothesis, we found that, as in normal humans and dogs (17), the plasma lactate level increased rapidly but transiently following an i.v. glucose challenge in WT mice (Figure 4, A and B) and that this effect was blunted in vehicle-treated $o b / o b$ mice (Figure 4, $\mathrm{A}$ and $\mathrm{B})$. Indeed, lactate levels did not increase at all in these animals, whereas in $o b / o b$ mice receiving i.c.v. FGF19, the plasma lactate response to i.v. glucose increased markedly (Figure 4, A and B), as did GE (Figure 3).

To determine whether the effect of FGF19 to increase GE involves reductions of either plasma glucagon or nonesterified fatty acid (FFA) levels, we perf utes: $340 \pm 51 \mathrm{mg} / \mathrm{dl}$ for vehicle vs. $241 \pm 34 \mathrm{mg} / \mathrm{dl}$ for FGF19; $P<0.05)$. However, the glucose-lowering effect of FGF19 was not associated with significant changes in the plasma level of either glucagon or FFAs (Figure 4, C and D).

Liver parameters. As a first step to investigate whether changes of liver glucose metabolism contributed to the glucose-lowering effect of i.c.v. FGF19, we measured baseline liver glycogen and triglyceride content as well as hepatic expression of gluconeogenic 
A
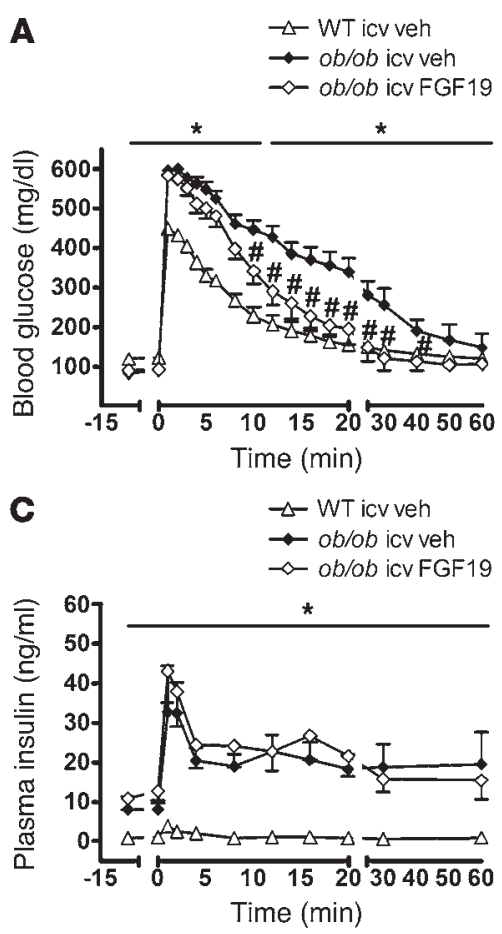

E

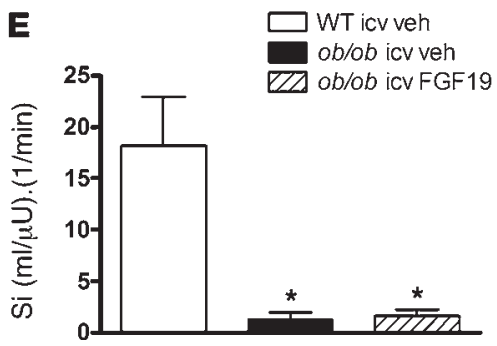

B
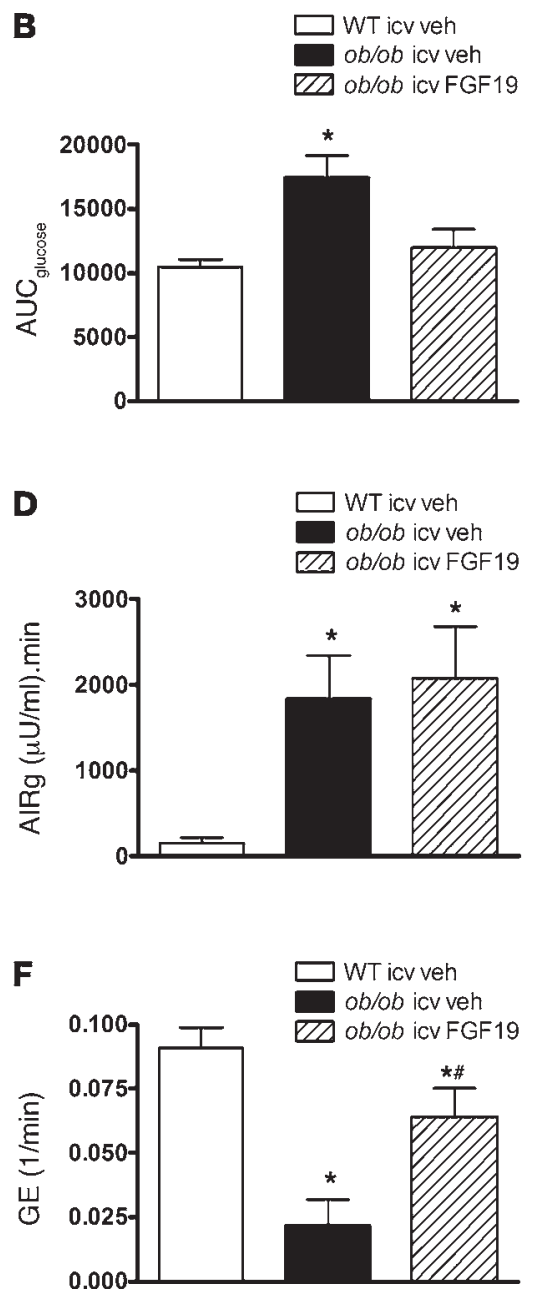

Figure 3

Effect of FGF19 on determinants of glucose tolerance in ob/ob mice. (A) Blood glucose, (B) the integrated area under the glucose curve, (C) plasma insulin levels, (D) the acute insulin response to glucose (AIRg), (E) $\mathrm{Si}$, and (F) GE in ob/ob mice or littermate controls that received either i.c.v. vehicle or FGF19 $(3 \mu \mathrm{g})$ and were subjected to a frequently sampled glucose tolerance test. Data represent mean \pm SEM. ${ }^{*} P<0.05$ vs. WT i.c.v. vehicle; ${ }^{\#} P<0.05$ vs. ob/ob i.c.v. vehicle. genes in the absence of a glucose challenge. Our data show that while hepatic levels of G6Pase were significantly reduced in $o b / o b$ mice treated with i.c.v. FGF19 relative to vehicle-treated $o b / o b$ and WT controls, there were no differences in hepatic Pepck mRNA levels or in either liver glycogen or triglyceride content (Figure 5).

To determine whether the effect of i.c.v. FGF19 to enhance disposal of a glucose load is associated with increased liver glycogen content, as expected if the mechanism involves increased liver glucose uptake and glycogen synthesis, we measured liver glycogen content 30 minutes after an i.p. glucose bolus $(0.5 \mathrm{~g} / \mathrm{kg})$ in $o b / o b$ mice that had received i.c.v. injection of either vehicle or FGF19 $(3 \mu \mathrm{g}) 2$ hours earlier. Consistent with our previous observations, central FGF19 administration reduced blood glucose levels relative to those of vehicle-treated controls both at baseline and $30 \mathrm{~min}$ utes after the glucose bolus (baseline: $179 \pm 31 \mathrm{mg} / \mathrm{dl}$ for vehicle vs. $106 \pm 26 \mathrm{mg} / \mathrm{dl}$ for FGF19; $P<0.05 ; 30$ minutes: $330 \pm 46 \mathrm{mg} / \mathrm{dl}$ for vehicle vs. $243 \pm 5 \mathrm{mg} / \mathrm{dl}$ for FGF19; $P<0.05)$. However, the glucose-lowering effect of FGF19 was not associated with a significant increase in hepatic glycogen levels $(8.83 \pm 0.57 \mathrm{mg} / \mathrm{g}$ tissue for vehicle vs. $8.60 \pm 1.17 \mathrm{mg} / \mathrm{g}$ tissue for FGF19; $P=\mathrm{NS}$ ). We next examined whether hepatic glycogen levels are increased following an i.p. glucose bolus in $o b / o b$ animals that receive systemic (rather than central) FGF19 (1 mg/kg i.p.). Again, the glucose-lowering effect of FGF19 was not associated with a significant change of hepatic glycogen levels $(9.66 \pm 0.93 \mathrm{mg} / \mathrm{g}$ tissue for vehicle vs. 9.37 $\pm 0.37 \mathrm{mg} / \mathrm{g}$ tissue for FGF19; $P=\mathrm{NS}$ ). These results indicate that, in $o b / o b$ mice, the effect of FGF19 to improve glucose tolerance, selectively increase GE, and raise plasma lactate levels occurs despite no change in liver glycogen content.

Role of melanocortin signaling in glucoregulatory effects of i.c.v. FGF19. As the melanocortin pathway is implicated in central regulation of glucose metabolism by leptin $(18,19)$, insulin $(20,21)$, and glucose, we asked whether (a) FGF19 activates proopiomelanocortin (POMC) neurons in the arcuate nucleus (as judged by induction of c-Fos staining) and (b) whether intact melanocortin signaling is required for the glucose-lowering effect of i.c.v. FGF19. To facilitate detection of c-Fos immunoreactivity in POMC neurons, we studied the effect of i.c.v. FGF19 in C57BL/6 mice in which POMC cells are fluorescently labeled (Pomc-Tau-GFP mice). As depicted in Figure 6, there was no effect of i.c.v. FGF19 to induce c-Fos in labeled POMC cells relative to i.c.v. vehicle-treated controls.

To determine whether melanocortin signaling is required for the glucose-lowering effects of central FGF19, we asked whether it lowers fasting blood glucose levels or improves glucose tolerance in melanocortin-4 receptor-deficient (Mc4r-deficient) mice. Indeed, i.c.v. FGF19 clearly exerts a glucose-lowering action in these animals relative to that in i.c.v. vehicle-treated animals $(P<0.05)$ (Figure 6, C and D). Therefore, 

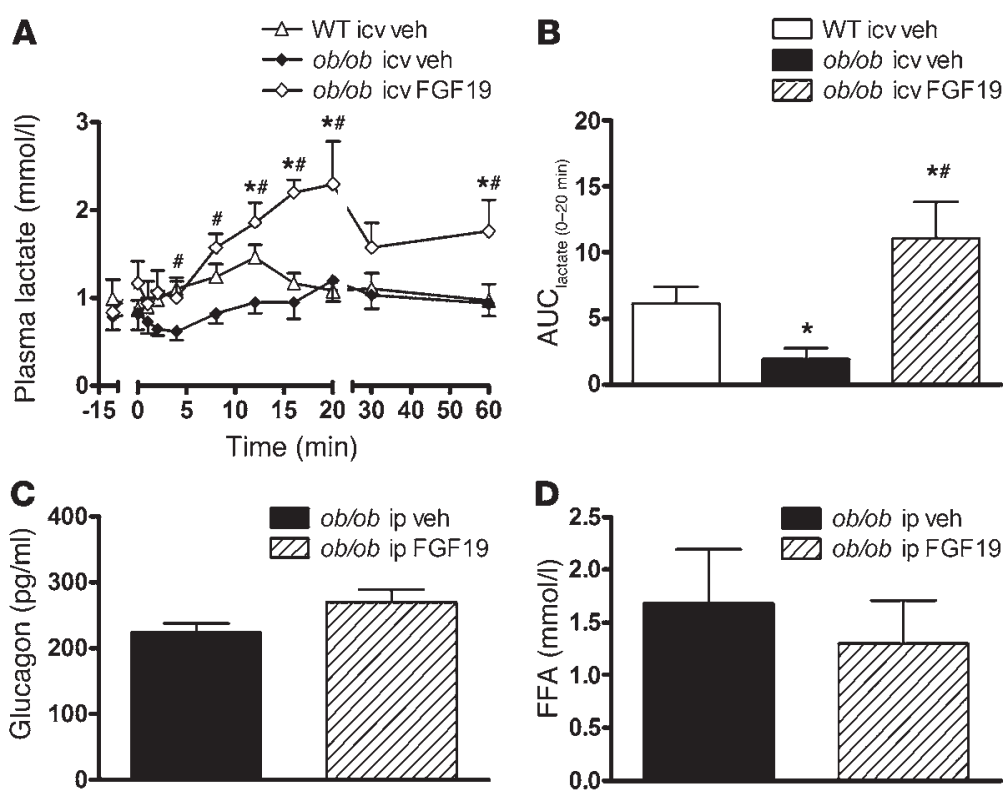

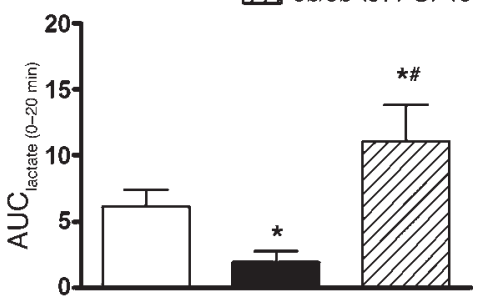

D

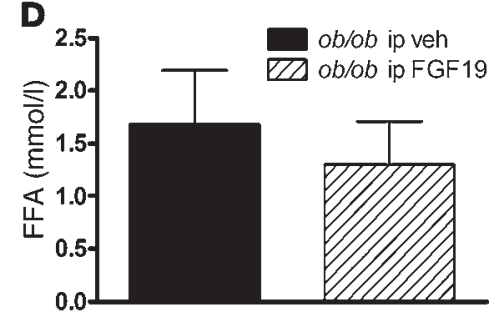

\section{Figure 4}

Effect of FGF19 on plasma glucagon, FFA, and lactate levels in ob/ob mice. (A) Plasma lactate levels and (B) the integrated area under the lactate curve ( $\left.\mathrm{AUC}_{\text {lactate }}\right)$ during the first 20 minutes of the same FSIGT performed in WT and ob/ob mice that received either i.c.v. vehicle or FGF19 shown in Figure 3. (C) Plasma glucagon and (D) FFA levels in ob/ob mice treated with FGF19 (1 $\mathrm{mg} / \mathrm{kg}$ i.p.) or its vehicle, followed 90 minutes later by an i.p. glucose bolus. Glucagon and FFA levels were measured in plasma obtained 30 minutes after the glucose bolus. Data represent mean \pm SEM. ${ }^{*} P<0.05$ vs. WT i.c.v. vehicle; ${ }^{\#} P<0.05$ vs. ob/ob i.c.v. vehicle.
FGF19-induced glucose lowering is mediated at least in part via a melanocortin-independent mechanism.

\section{Discussion}

Observations made in a rat model of uncontrolled, insulindeficient diabetes suggest that the brain has the capacity to lower blood glucose levels via insulin-independent means. Specifically, continuous or repeated daily i.c.v. leptin administration can normalize elevated blood glucose levels in these animals despite persistent, severe insulin deficiency (22-25). Building upon this initial finding, we hypothesized that, since the insulin-independent component of glucose tolerance, GE, is markedly reduced in $o b / o b$ mice, this defect will be remedied by FGF19 (2), because it has potent antidiabetic effects in these animals. We further hypothesized that since FGF19 is effective following either peripheral or low-dose central administration in these animals (6), its effect to increase GE is mediated centrally. Our finding that glucose lowering induced by systemic FGF19 administration in $o b / o b$ mice is attenuated by central administration of an inhibitor of FGF receptors implicates the brain in this effect. Moreover, centrally administered FGF19 improves glucose tolerance in these animals via a mechanism that cannot be explained by changes of insulin secretion or insulin action but arises instead from a 3-fold increase of GE. Although a detailed understanding of mechanisms mediating this effect awaits further study, our data support a model in which central FGF19 increases GE by stimulating glycolysis of glucose to lactate and in which the neuronal systems involved are at least partially independent of melanocortin signaling. Taken together, these data implicate the CNS in the glucose-lowering effect of FGF19 and, to our knowledge, are the first to establish the ability of the brain to improve glucose tolerance by rapidly, potently, and selectively increasing insulin-independent glucose disposal. These findings therefore establish the existence of a novel brain mechanism for insulin-independent control of blood glucose levels.

Growing evidence suggests that the CNS plays a key role in the regulation of glucose homeostasis. In obese rodents, increased hypothalamic input from hormonal (i.e., insulin and leptin) or nutrient-related (i.e., FFA) signals improves hepatic Si via a mechanism involving vagal input to the liver, whereas disruption of this hypothalamic input causes insulin resistance (26-30). In addition to these effects, the recent finding that continuous i.c.v. infusion of leptin completely normalizes glycemia in rats with streptozotocin-induced diabetes (22-25) suggests that the brain has the capacity to potently lower glucose levels via mechanisms that are insulin independent. To investigate the hypothesis that FGF19 has the capacity to lower blood glucose by mechanisms that are insulin independent, we used minimal model analysis of glucose and insulin data from a FSIGT, an approach developed in humans and recently validated for use in mice (2). We found that, in $o b / o b$ mice, a single i.c.v. injection of a low dose of FGF19 improved glucose tolerance markedly, despite having no effect on either insulin secretion or action. The dramatic effect of centrally administered FGF19 to increase GE is remarkable, in that with the exception of vigorous exercise (31), there is no precedent for rapid upregulation of insulin-independent glucose disposal, much less by the brain.

The brain's inherent capacity to promote glucose lowering through insulin-independent mechanisms is novel in the sense that previous work in this area has focused largely on hypothalamic control of hepatic Si $(32,33)$. That insulin-independent effects were not identified in such studies could reflect the fact that the methods used are not optimized to detect them. The euglycemic, hyperinsulinemic clamp method, widely accepted as the "gold standard" for quantitative assessment of glucose metabolism and Si, has been used extensively in human as well as rodent studies. By comparison, although minimal model analysis of FSIGT data has seen wide usage in human research, it was only validated for use in mice in the last year (2). This method measures not only Si and GE, but also firstphase insulin secretion and the disposition index (the product of $\mathrm{Si}$ and insulin secretion, a validated predictor of progression to T2D in humans). By comparison, the clamp (but not the minimal model analysis) quantifies rates of glucose production and utilization and, with some modification, can assess rates of glycogenolysis, gluconeogenesis, and glucose uptake into specific (nonhepatic) tissues. 
A

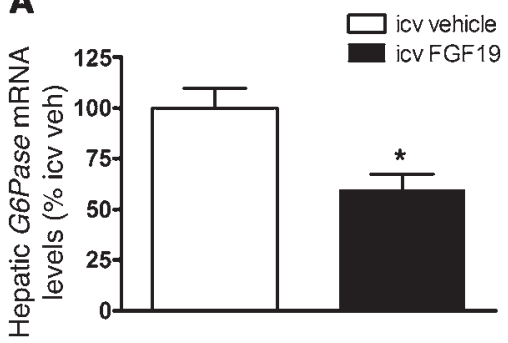

C

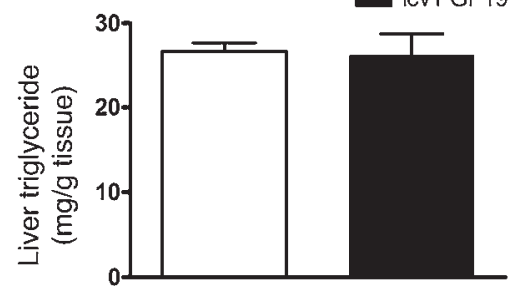

B

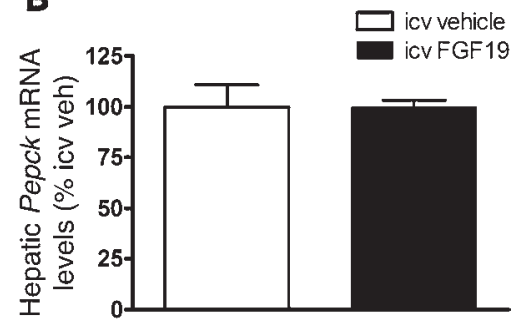

D
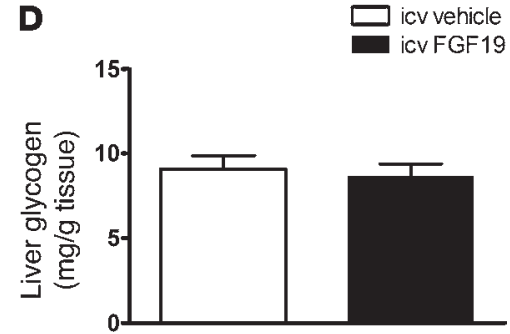

\section{Figure 5}

Hepatic effects of i.c.v. FGF19 in ob/ob mice. Hepatic expression of (A) G6Pase and (B) Pepck using real-time $\mathrm{PCR}$ and (C) liver triglyceride and (D) liver glycogen content in leptin-deficient $o b / o b$ animals that received daily i.c.v. injections of either FGF19 $(3 \mu \mathrm{g})$ or its vehicle. Data represent mean \pm SEM. ${ }^{*} P<0.05$ vs. i.c.v. vehicle.
Si is defined in clamp studies as the increase of glucose disposal (or decrease of glucose appearance) observed when insulin levels are raised while glucose levels are held constant. Stated differently, changes in rates of glucose production or disposal measured in the face of high insulin are attributed solely to the action of insulin. However, in the event that insulin-independent glucose disposal (or insulin-independent suppression of hepatic glucose production) is also increased by the intervention under study, the extent to which observed changes are attributable to insulin action cannot be known with certainty, since some of the glucose infused during the clamp (to maintain euglycemia in the presence of high insulin) would have been disposed of via insulin-independent mechanisms. Thus, whether insulin-independent mechanisms contributed to some of the centrally mediated effects attributed previously to changes of $\mathrm{Si}$ $(32,33)$ awaits additional study.

The insulin-independent component of glucose disposal in sedentary subjects has historically been considered to be a fixed, obligate, and invariant means whereby insulin-independent tissues obtain glucose primarily via the process of glucose mass action, by which increasing concentrations of glucose passively drive glucose into cells in a largely unregulated manner (1). It is for this reason that research into mechanisms underlying insulin-independent glucose disposal has languished in comparison with the substantial effort and progress made toward understanding insulin secretion and its action in peripheral tissues. Nevertheless, data from clinical studies performed years ago offer evidence suggestive of regulation. For one, i.v. infusion of glucagon-like peptide-1 (GLP-1) in humans improves i.v. glucose tolerance by increasing GE as well as insulin secretion (34), while, conversely, GE was reduced following i.v. administration of a somatostatin analog, an effect that impairs glucose tolerance beyond what can be explained by the associated inhibition of insulin secretion (35). Although the mechanism underlying these effects on GE is unknown, our data with FGF19 raise the possibility of an action mediated in the brain.

Although our conclusions regarding the role of GE in the antidiabetic effect of FGF19 are based primarily on studies conducted in $o b / o b$ mice, we believe that these findings have broader implications. First, our findings are compatible with several studies showing that, in rodent models of severe insulin-deficient diabetes, leptin administration can fully normalize blood glucose levels, despite persistent insulin deficiency (22-25). The effect is therefore insulin independent, and, moreover, it is mediated centrally, since the effect is observed when leptin is given i.c.v. at a much lower dose than is required during systemic administration. Combined with the current studies, these observations establish brain's inherent capacity to induce dramatic, insulin-independent glucose lowering and provide a compelling rationale for investigation into underlying mechanisms and their clinical relevance. The question of whether centrally active mediators other than leptin and FGF19 can increase GE is also relevant. For example, FGF21 exerts antidiabetic effects analogous to those induced by FGF19, and both hormones appear to signal via the same receptor (FGFR1), but whether FGF21 increases GE is unstudied. In addition to candidate peptides, an increase of circulating glucose levels is itself well known to increase GE, and the possibility that this involves an effect of glucose in the brain is consistent with available data (36).

The contribution made by insulin-independent mechanisms to overall glucose homeostasis is surprisingly large. Available evidence suggests that GE accounts for approximately $50 \%$ of glucose disposal in humans following an i.v. glucose challenge (in rodents, the contribution may be greater), with the remaining 50\% attributable to the combined effects of insulin action and insulin secretion (1). It is noteworthy in this context that, like insulin resistance, reduced GE makes an important contribution - perhaps comparable to that of insulin resistance - to impaired glucose tolerance and T2D in humans $(1,37)$. Consequently, reduced GE is a strong risk factor for the development of T2D (38). Combined with our current findings, the possibility that a central defect contributes to impaired GE in obesity-associated metabolic impairment can be considered. Related questions of strong translational interest include whether defects in CNS sensing or responsiveness to FGF19 or other inputs contribute to common clinical disorders of glucose homeostasis and whether interventions that increase GE have therapeutic potential in individuals with obesity and diabetes.

As a first step to investigate how FGF19 action in the brain increases GE, we measured the increment of plasma lactate 

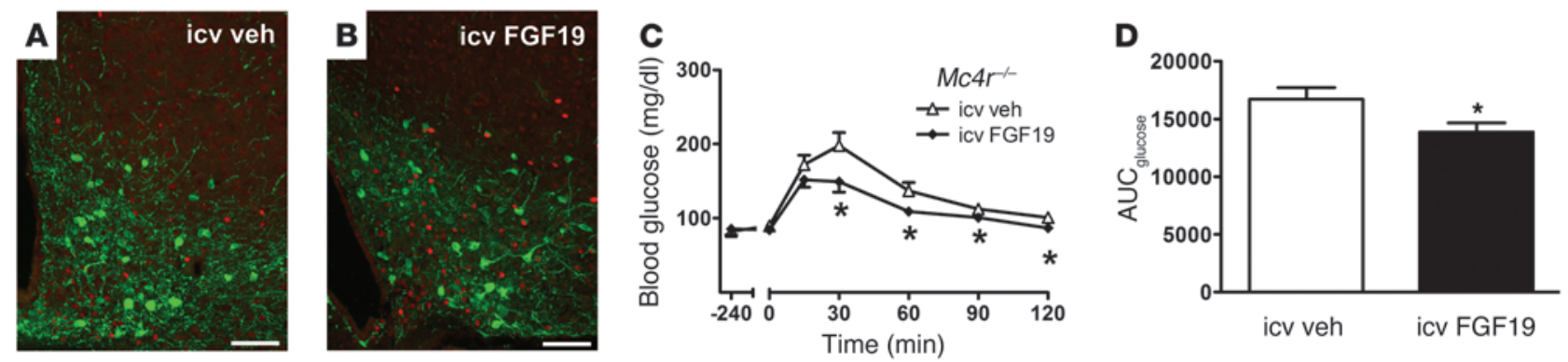

\section{Figure 6}

Central targets of FGF19 action. (A and B) Immunodetection of POMC (green) and the colocalization of c-fos in POMC neurons in Pomc-Tau-GFP mice treated with i.c.v. administration of either (A) vehicle or (B) FGF19 (original magnification, $\times 20$; Scale bar: $20 \mu \mathrm{m}$ ). (C) Glucose tolerance $(0.5 \mathrm{~g} / \mathrm{kg})$ and (D) the integrated area under the glucose curve in $M c 4 r^{-1}$-mice that received a single, acute i.c.v. injection of FGF19 (3 $\left.\mu \mathrm{g}\right)$ or its vehicle. Data represent mean $\pm \mathrm{SEM}$. ${ }^{*} P<0.05$ vs. i.c.v. vehicle. $3 \mathrm{~V}$, third ventricle.

levels that follows a glucose challenge. The rationale for this approach begins with evidence published some 40 years ago that plasma lactate levels increase following consumption of a mixed meal in humans (39). Subsequent studies showed that this response results not from lactate absorption from the GI tract, but from the uptake of glucose into tissues, followed by its metabolism to lactate that is subsequently released into the circulation $(40,41)$. Since the same glucose $\rightarrow$ lactate metabolic response occurs after an i.v. glucose challenge, calories do not need to enter the body from the GI tract to elicit this response - an increased circulating glucose level is sufficient (15). Interestingly, the effect of either oral or i.v. glucose to increase lactate levels is reduced in obese humans (15), as is GE (1). We therefore asked (a) whether reduced GE in $o b / o b$ mice is associated with blunting of the glucose $\rightarrow$ lactate response and (b) whether FGF19 action in the brain increases GE in part by stimulating this process. Our data are consistent with both predictions. We found that the plasma lactate level increased rapidly following an i.v. glucose challenge in WT mice, whereas lactate levels did not increase at all in vehicle-treated $o b / o b$ mice, consistent with their markedly reduced GE (by $\sim 70 \%$ ). In contrast, both GE and the plasma lactate response to i.v. glucose increased markedly in $o b / o b$ mice receiving i.c.v. FGF19. Together, these findings support a model in which (a) reduced GE in $o b / o b$ mice results in part from a decreased glucose $\rightarrow$ lactate metabolic response, (b) the effect of FGF19 to increase GE involves an increase in this response, and (c) the brain mediates this effect of FGF19.

The identification of tissues in which this glucose $\rightarrow$ lactate response occurs in mice awaits further study. In dogs and humans, the liver is implicated, and it is notable in this context both that liver glucose uptake is insulin independent and that the brain plays a fundamental role to regulate this process (38). However, our finding that the effect of FGF19 to increase GE is not associated with increased liver glycogen content in $o b / o b$ mice raises questions regarding whether liver glucose uptake is increased, and both fat and skeletal muscle can also metabolize glucose to produce lactate that is subsequently released into the circulation $(42,43)$. Additional studies are therefore needed to definitively identify the tissue(s) involved.

Although our findings support the hypothesis that the antidiabetic effects of FGF19 involve a central site of action, we empha- size that they do not preclude a role for peripheral tissue effects as well $(8,44)$. FGFR1 activation exerts glucose-lowering effects in liver as well as both brown and white adipose tissue (11), and the beneficial effects of systemic FGF19 on glucose tolerance that we observed in $o b / o b$ mice were only partially blocked by central FGF receptor blockade. Future studies using targeted disruption of FGF receptor signaling in a tissue-specific manner will help to clarify the extent to which glucoregulatory effects of FGF19 are mediated centrally versus peripherally.

In addition, little is known regarding the neurocircuitry underlying central FGF19 action, although our data implicate a mechanism independent of melanocortin signaling. This assertion is based on our findings that FGF19 failed to induce c-Fos (a marker of neuronal activation) in hypothalamic POMC neurons and that FGF19 improved glucose tolerance in Mc4r-deficient mice (albeit, not to the same extent as was observed in $o b / o b$ mice). Whatever the underlying neurocircuitry, convergent lines of evidence that implicate activation of FGFR1 in the antidiabetic effects of FGF19 (and FGF21, as well) $(9,10)$ and the observation that FGFR1 is expressed in key hypothalamic areas implicated in metabolic regulation $(12,13)$ support a model in this brain area that mediates the increase of GE induced by FGF19. Additional studies are warranted to test this hypothesis.

Whether FGF19 plays a physiological role in glucose homeostasis or diabetes pathogenesis is still largely unstudied. Also of interest is the as yet untested hypothesis that beneficial metabolic effects of bariatric surgical procedures are tied to increased FGF19 secretion from gut enterocytes. Consistent with this possibility, circulating levels of bile acids $(45,46)$ and FGF19 increase following roux-en-Y gastric bypass (RYGB) surgery in humans $(47,48)$. As bile acid stimulation of FGF19 secretion is mediated via FXR, these data collectively raise the possibility that increased bile acid-induced stimulation of FGF19 secretion (and increased GE) contributes to the high rate of diabetes remission induced by RYGB (49), but a direct test of this hypothesis is still awaited.

In conclusion, our findings suggest that the glucose-lowering effect of systemic FGF19 involves a novel central mechanism whereby insulin-independent glucose disposal is rapidly, potently, and selectively increased. The finding that the brain has the inherent ability to lower blood glucose levels via such a mechanism opens new potential avenues for diabetes drug discovery. 


\section{Methods}

Animals. All animals were housed individually under specific pathogen-free conditions in a temperature-controlled room with a 12-hour light/12-hour dark cycle. Animals were provided with ad libitum access to water and standard laboratory chow (PMI Nutrition International Inc.) unless otherwise stated. Adult, male $o b / o b$ mice on the C57/B16 background and their WT littermates were obtained from The Jackson Laboratory. To better identify POMC cells, we used Pomc-Tau-eGFP mice that were provided to us by Tomas Horvath at Yale University (New Haven, Connecticut, USA), in which T-topaz GFP is expressed under the transcriptional control of the POMC promoter and backcrossed onto the $\mathrm{C} 57 / \mathrm{Bl} 6$ background for $>10$ generations (50). $\mathrm{Mc}^{4} \mathrm{r}^{-1-}$ mice, originally derived as discussed in ref. 51 , were obtained from breeding colonies maintained at the University of Washington.

Surgery. Animals underwent cannulation of the lateral ventricle (Plastics One) under isoflurane anesthesia at stereotaxic coordinates based on the brain atlas of Paxinos and Watson (52): $-0.7 \mathrm{~mm}$ posterior to bregma; $1.3 \mathrm{~mm}$ lateral, and $1.3 \mathrm{~mm}$ below the skull surface. The cannula was permanently fixed to the skull using glass ionomer cement (Instech Solomon) and was closed with an obturator. Animals received buprenorphine hydrochloride (Reckett Colman Pharmaceuticals) at the completion of the surgery and were allowed to recover for at least 5 days while food intake and body weight were recorded. i.c.v. injections were administered using a (33-gauge) needle that extended $1 \mathrm{~mm}$ beyond the tip of the cannula over a period of 60 seconds in a final volume of up to $1.5 \mu l$. The injector was then allowed to remain in place for an addition 60 seconds before removal. For studies involving a FSIGT, adult male C57/B16 and ob/ob mice underwent lateral ventricular cannulation as above as well as catheterization of both the carotid artery and jugular vein, as described in detail previously (53).

Body composition analysis. Liver triglyceride content and measures of total fat and lean body mass were determined using quantitative magnetic resonance spectroscopy (EchoMRI 3-in-1 Animal Tissue Composition Analyzer; Echo Medical Systems) using the Energy Balance and Glucose Metabolism Core of the Nutrition Obesity Research Center at the University of Washington. Liver glycogen levels were determined using a colorimetric assay (Biovision) and were standardized to grams wet weight.

i.p. glucose tolerance testing. i.p. glucose tolerance tests (ipgtt; $30 \%$ D-glucose; 0.5 or $1.5 \mathrm{~g} / \mathrm{kg}$ ) were conducted in 5 -hour fasted animals. Blood glucose levels were measured at $t=0,15,30,60,90$, and 120 minutes using a hand-held glucometer (Accu-Chek) to test blood obtained from tail capillary samples.

CNS FGF receptor signaling in the glucose-lowering effects of FGF19 in $o b / o b$ mice. To determine whether increased FGF19 signaling in the brain is sufficient to improve glucose tolerance, adult male $o b / o b$ mice were matched for body weight, food intake, and blood glucose levels and fasted at 09:00. At 11:30, animals received a single, acute i.c.v. injection of either vehicle ( $0.9 \%$ saline) or FGF19 (Phoenix Pharmaceuticals) at a dose of $1 \mu \mathrm{g}$ or $3 \mu \mathrm{g}$. At 13:00, animals were subjected to an ipgtt $(0.5 \mathrm{~g} / \mathrm{kg})$. Animals followed the same paradigm as described above, except that they received an i.p. injection of FGF19 at the same doses as administered i.c.v.

To determine whether the glucose-lowering effect of FGF19 involves activation of FGF receptors in the CNS, a separate cohort of $o b / o b$ mice was matched for body weight, food intake, and blood glucose levels and fasted at 09:00. Ninety minutes later, animals received either an i.c.v. preinjection of the FGFR inhibitor, PD173074 (Sigma-Aldrich; $25 \mu \mathrm{g}$ ), or its vehicle (100\% DMSO). Relative to vehicle-treated controls, this dose had no effect by itself on either fasting blood glucose levels or glucose tolerance (data not shown). To confirm that the glucose-lowering effect of i.c.v. FGF19 is receptor mediated, animals received an i.c.v. injection of
FGF19 $(3 \mu \mathrm{g})$ or its vehicle 1 hour after pretreatment injection. At 13:00, animals were then subjected to an ipgtt $(0.5 \mathrm{~g} / \mathrm{kg})$. Once the efficacy of the inhibitor was validated against i.c.v. FGF19, we asked whether pretreatment blunts the antidiabetic effect of FGF19 following systemic administration at a higher dose. Adult male $o b / o b$ mice followed the same protocol as described above, except that they received a systemic (rather than i.c.v.) injection of FGF19 (1 mg/kg).

To determine whether the glucose-lowering effects of i.c.v. FGF19 require melanocortin signaling, adult male $\mathrm{Mc} \mathrm{r}^{-/}$- mice bred onto the C57BL/6 background and matched for age, body weight, and blood glucose were subjected the above protocol, in which an ipgtt $(1.5 \mathrm{~g} / \mathrm{kg})$ was performed 90 minutes following i.c.v. injection of FGF19 $(3 \mu \mathrm{g})$ or its vehicle.

Effect of chronic i.c.v. FGF19 on blood glucose levels in ob/ob mice. To determine whether chronic i.c.v. administration of FGF19 ameliorates diabetes in $o b / o b$ mice, adult male $o b / o b$ mice matched for body weight, food intake, and blood glucose levels received daily i.c.v. injections of FGF19 $(3 \mu \mathrm{g})$ or its vehicle for 5 consecutive days. Body weight, food intake, and blood glucose levels were recorded daily. At the completion of the study, animals were perfused, and brains removed and processed for immunohistochemical outcomes, as described below. To determine whether the glucose-lowering effects of CNS FGF19 could be explained by leakage into the periphery, a similar paradigm was used, except that $o b / o b$ mice received daily peripheral injections of FGF19 $(3 \mu \mathrm{g})$ or its vehicle at the same dose that was administered i.c.v.

FSIGT. A FSIGT was performed in 5-hour fasted $o b / o b$ mice or their littermate controls at least 5 days following LV cannulation and arterial and venous catheterization. Blood sampling was performed via the arterial catheter in unrestrained, conscious animals. A baseline fasted blood sample was taken at -10 and 0 minutes. Based on a previous protocol (2), a $1 \mathrm{~g} / \mathrm{kg}$ bolus of $50 \%$ dextrose was injected i.v. over a period of 15 seconds at $t=0$ minutes. Blood $(20 \mu \mathrm{l})$ was sampled for measurement of glucose and subsequent assay of plasma insulin at time points $1,2,4,8,12,16,20,30$, and 60 minutes. Additional samples were obtained for glucose measurement alone at $3,5,6,10,14,18,25,40$, and 50 minutes. Throughout the entire procedure, beginning at -10 minutes, mice received i.v. infusion of salinewashed erythrocytes $(5 \mu \mathrm{l} / \mathrm{min})$ to prevent the significant fall in hematocrit that would otherwise occur. Blood glucose levels were measured on a handheld glucometer (Accu-Chek).

Minimal model analysis of FSIGT data. Based on glucose and insulin curves, glucose disposal was divided into 3 phases: mixing phase (0-2 minutes), GE-dominated phase (2-5 minutes), and insulin-dominated phase (5-60 minutes) (2). The mixing phase was not used for $\mathrm{Si}$ or GE modeling. Acute insulin response to glucose was based on insulin values between 0 and 4 minutes. All Si calculations are multiplied by 104 . Data were modeled using MinMod software modified for phase durations as stated above and GE starting estimate.

Effect of i.c.v. FGF19 on c-fos induction in Pomc-Tau-eGFP mice. To determine whether FGF19 activates POMC neurons in the hypothalamus, female Pomc-Tau-eGFP mice underwent lateral ventricular cannulation, as described previously. Following a 1-week recovery period, habituated animals were fasted for 3 -hours and then received an i.c.v. injection of either saline or FGF19 (3 $\mu \mathrm{g})$. Ninety minutes later, mice were perfused, with brains removed and processed for immunohistochemical outcomes, as described below.

Tissue collection and processing. For immunohistochemical studies, mice were anesthetized with ketamine/xylazine and perfused with PBS followed by $4 \%$ PFA in $0.1 \mathrm{M}$ PBS. Brains were removed, post-fixed in $4 \%$ PFA overnight, embedded in $25 \%$ sucrose solution for 48 hours at $4{ }^{\circ} \mathrm{C}$, snap frozen in isopentane, and cooled with liquid nitrogen. The hypothalamus was sectioned at $14 \mu \mathrm{M}$ in the coronal plane using a freezing cryotome, slide- 
mounted, and stored at $-80^{\circ} \mathrm{C}$ until selection for immunohistochemical staining or visualization of GFP by fluorescence microscopy.

Double-label immunofluorescence. For colocalization studies, anatomically matched sections throughout the hypothalamus were selected for staining. Briefly, slides were washed with $10 \mathrm{mM}$ PBS at room temperature and were then blocked for 30 minutes with buffer containing 5\% normal donkey serum, $0.1 \%$ BSA, and $0.01 \%$ Triton- $\mathrm{X}$ in $10 \mathrm{mM}$ PBS. Following additional washes, slides were incubated overnight at $4{ }^{\circ} \mathrm{C}$ in a "cocktail" of primary antibodies, including goat polyclonal anti-GFP (Fitzgerald Industries) diluted 1:10,000 with rabbit polyclonal anti-c-Fos (Oncogene Research Products) diluted 1:100,000. Slides were washed with PBS and then incubated for 120 minutes at room temperature with the secondary antibodies (a) 1:1,000 donkey anti-goat IgG-Alexa Fluor 488 (Life Technologies) and (b) 1:1,000 donkey anti-rabbit Alexa Fluor 594 (Life Technologies), diluted in 5\% normal donkey serum and $0.1 \%$ BSA in $10 \mathrm{mM}$ PBS. After washing with PBS, slides were subsequently coverslipped with an antifade aqueous mounting media. Control sections were incubated with normal serum and did not show staining.

Plasma analysis. Blood samples for measures of insulin, lactate, and FFAs were collected in EDTA-treated tubes, while blood for glucagon assay was collected on tubes containing $10 \mu \mathrm{l}$ of $1 \mathrm{M}$ benzamidine and $1 \mathrm{U}$ of heparin. Blood was centrifuged and plasma was removed, aliquoted, and stored at $-80^{\circ} \mathrm{C}$. Plasma immunoreactive insulin levels were determined by ELISA (Crystal Chem), FFA levels were determined using a colorimetric assay (Wako Chemicals), plasma lactate levels were determined using a GM9D glucose direct analyzer (Analox Instruments), and plasma glucagon levels were determined using a glucagon RIA kit (Linco Research).

RT-PCR. Total RNA was extracted from liver using TRIzol B according to manufacturers' instructions (MRC). RNA was quantitated by spectrophotometry at $260 \mathrm{~nm}$ (Nanodrop 1000, Thermo Scientific) and reverse-transcribed with AMV reverse transcriptase $(1 \mu \mathrm{g})$ (Promega). Realtime PCR was performed on a ABI Prism 7900 HT (Applied Biosystems) using the commercially available PCR Master Mix (SYBR Green, Applied 2.0; Applied Biosystems) and analyzed as previously described $(22,26)$.

Statistics. All results are expressed as mean \pm SEM. Statistical analyses were performed using Statistica (version 7.1). A 1-way analysis of variance with a LSD post-hoc test was used to compare mean values among multiple groups using log-transformed data when Bartlett's test for unequal variance showed $P<0.05$, and a 2 -sample unpaired Student's $t$ test was used for 2-group comparisons. In all instances, probability values of $<0.05$ were considered significant.

Study approval. All procedures were performed in accordance with NIH guidelines for the care and use of animals and were approved by the Institutional Animal Care and Use Committee at both the University of Washington and Vanderbilt University.

\section{Acknowledgments}

The authors acknowledge the technical assistance provided by Alex Cubelo and J.D. Fisher at the University of Washington. This work was supported by NIH grants DK083042 and DK090320 (to M.W. Schwartz) and DK089053 (to G.J. Morton), the NIDDKfunded Nutrition Obesity Research Center (NORC, DK035816) and Diabetes Research Center (DRC, DK017047) at the University of Washington, and the Mouse Metabolic Phenotyping Center at Vanderbilt University (MMPC, U24 DK059637).

Received for publication April 26, 2013, and accepted in revised form August 1, 2013.

Address correspondence to: Michael W. Schwartz, Department of Medicine, University of Washington at South Lake Union, 850 Republican St., N335, Box 358055, Seattle, Washington 98195, USA. Phone: 206.897.5288; Fax: 206.897.5293; E-mail: mschwart@u.washington.edu.
1. BestJD, Kahn SE, Ader M, Watanabe RM, Ni TC, Bergman RN. Role of glucose effectiveness in the determination of glucose tolerance. Diabetes Care. 1996 . 19(9):1018-1030.

2. Alonso LC, et al. Simultaneous measurement of insulin sensitivity, insulin secretion, and the disposition index in conscious unhandled mice. Obesity (Silver Spring). 2012;20(7):1403-1412.

3. Beenken A, Mohammadi M. The FGF family: biology, pathophysiology and therapy. Nat Rev Drug Discov. 2009;8(3):235-253.

4. Holt JA, et al. Definition of a novel growth factordependent signal cascade for the suppression of bile acid biosynthesis. Genes Dev. 2003;17(13):1581-1591.

5. Inagaki $\mathrm{T}$, et al. Fibroblast growth factor 15 functions as an enterohepatic signal to regulate bile acid homeostasis. Cell Metab. 2005;2(4):217-225.

6. Fu L, et al. Fibroblast growth factor 19 increases metabolic rate and reverses dietary and leptin-deficient diabetes. Endocrinology. 2004;145(6):2594-2603.

7. Tomlinson E, et al. Transgenic mice expressing human fibroblast growth factor-19 display increased metabolic rate and decreased adiposity. Endocrinology. 2002;143(5):1741-1747.

8. Kir S, et al. FGF19 as a postprandial, insulin-independent activator of hepatic protein and glycogen synthesis. Science. 2011;331(6024):1621-1624.

9. Huang X, Yang C, Luo Y, Jin C, Wang F, McKeehan WL. FGFR4 prevents hyperlipidemia and insulin resistance but underlies high-fat diet induced fatty liver. Diabetes. 2007;56(10):2501-2510.

10. Wu AL, et al. FGF19 regulates cell proliferation, glucose and bile acid metabolism via FGFR4-dependent and independent pathways. PLoS One. 2011; 6(3):e17868.
11. Wu AL, et al. Amelioration of type 2 diabetes by antibody-mediated activation of fibroblast growth factor receptor 1. Sci Transl Med. 2011; 3(113):113ra126.

12. Gonzalez AM, Berry M, Maher PA, Logan A, Baird A. A comprehensive analysis of the distribution of FGF-2 and FGFR1 in the rat brain. Brain Res. 1995; 701(1-2):201-226.

13. Matsuo A, et al. Immunohistochemical localization in the rat brain of an epitope corresponding to the fibroblast growth factor receptor-1. Neuroscience. 1994;60(1):49-66

14. Ryan KK, Kohli R, Gutierrez-Aguilar R, Gaitonde SG, Woods SC, Seeley RJ. Fibroblast growth factor-19 action in the brain reduces food intake and body weight and improves glucose tolerance in male rats. Endocrinology. 2013;154(1):9-15.

15. Lovejoy J, Newby FD, Gebhart SS, DiGirolamo $\mathrm{M}$. Insulin resistance in obesity is associated with elevated basal lactate levels and diminished lactate appearance following intravenous glucose and insulin. Metabolism. 1992;41(1):22-27.

16. Ho JE, et al. Metabolic profiles during oral glucose glucose challenge. Diabetes. 2013;62(8):2689-2698.

17. Stefanovski D, et al. Estimating hepatic glucokinase activity using a simple model of lactate kinetics. Diabetes Care. 2012;35:1015-1020.

18. da Silva AA, do Carmo JM, Freeman JN, Tallam LS, Hall JE. A functional melanocortin system may be required for chronic CNS-mediated antidiabetic and cardiovascular actions of leptin. Diabetes. 2009; 58(8):1749-1756.

19. Berglund ED, et al. Direct leptin action on POMC neurons regulates glucose homeostasis and hepatic insulin sensitivity in mice. J Clin Invest. 2012;
122(3):1000-1009.

20. Hill JW, et al. Direct insulin and leptin action on proopiomelanocortin neurons is required for normal glucose homeostasis and fertility. Cell Metab. 2010; 11(4):286-297.

21. Konner AC, et al. Insulin action in AgRP-expressing neurons is required for suppression of hepatic glucose production. Cell Metab. 2007;5(6):438-449.

22. German JP, et al. Leptin action in the brain normalizes diabetic hyperglycemia via insulin-independent mechanisms. Endocrinology. 2011; 152(5):394-404.

23. Fujikawa T, Chuang JC, Sakata I, Ramadori G, Coppari R. Leptin therapy improves insulin-deficient type 1 diabetes by CNS-dependent mechanisms in mice. Proc Natl Acad Sci U S A. 2010; 107(40):17391-17396.

24. Hidaka S, et al. Chronic central leptin infusion restores hyperglycemia independent of food intake and insulin level in streptozotocin-induced diabetic rats. FASEB J. 2002;16(6):509-518.

25. Lin CY, Higginbotham DA, Judd RL, White BD. Central leptin increases insulin sensitivity in streptozotocin-induced diabetic rats. Am J Physiol Endocrinol Metab. 2002;282(5):E1084-E1091.

26. German JP, et al. Hypothalamic leptin signaling regulates hepatic insulin sensitivity via a neurocircuit involving the vagus nerve. Endocrinology. 2009; 150(10):4502-4511

27. Lam TK, et al. Hypothalamic sensing of circulating fatty acids is required for glucose homeostasis. Nat Med. 2005;11(3):320-327.

28. Obici S, Feng Z, Morgan K, Stein D, Karkanias G, Rossetti L. Central administration of oleic acid inhibits glucose production and food intake. Dia- 
betes. 2002;51(2):271-275.

29. Obici S, Zhang BB, Karkanias G, Rossetti L. Hypothalamic insulin signaling is required for inhibition of glucose production. Nat Med. 2002; 8(12):1376-1382

30. Pocai A, et al. Hypothalamic K(ATP) channels control hepatic glucose production. Nature. 2005; 434(7036):1026-1031.

31. Kahn SE, et al. Exercise training delineates the importance of B-cell dysfunction to the glucose intolerance of human aging. J Clin Endocrinol Metab. 1992;74(6):1336-1342.

32. Lam CK, Chari M, Lam TK. CNS regulation of glucose homeostasis. Physiology (Bethesda). 2009; 24:159-170.

33. Morton GJ, Schwartz MW. Leptin and the central nervous system control of glucose metabolism. Physiol Rev. 2011;91(2):389-411.

34. D'Alessio DA, Kahn SE, Leusner CR, Ensinck JW. Glucagon-like peptide 1 enhances glucose tolerance both by stimulation of insulin release and by increasing insulin-independent glucose disposal. J Clin Invest. 1994;93(5):2263-2266.

35. Kahn SE, et al. Treatment with a somatostatin analog decreases pancreatic B-cell and whole body sensitivity to glucose. J Clin Endocrinol Metab. 1990; 71(4):994-1002.

36. Lam TK, Gutierrez-Juarez R, Pocai A, Rossetti L. Regulation of blood glucose by hypothalamic pyruvate metabolism. Science. 2005;309(5736):943-947.

37. Taniguchi A, et al. Insulin secretion, insulin sensi- tivity, and glucose effectiveness in nonobese individuals with varying degrees of glucose tolerance. Diabetes Care. 2000;23(1):127-128.

38. Martin BC, Warram JH, Krolewski AS, Bergman RN, Soeldner JS, Kahn CR. Role of glucose and insulin resistance in development of type 2 diabetes mellitus: results of a 25 -year follow-up study. Lancet. 1992;340(8825):925-929.

39. Felig P, Wahren J, Hendler R. Influence of oral glucose ingestion on splanchnic glucose and gluconeogenic substrate metabolism in man. Diabetes. 1975; 24(5):468-475.

40. Davis MA, Williams PE, Cherrington AD. Effect of a mixed meal on hepatic lactate and gluconeogenic precursor metabolism in dogs. Am J Physiol. 1984; 247(3 pt 1):E362-E369.

41. Davis MA, Williams PE, Cherrington AD. Net hepatic lactate balance following mixed meal feeding in the four-day fasted conscious dog. Metabolism. 1987; 36(9):856-862.

42. Brooks GA. Lactate production under fully aerobic conditions: the lactate shuttle during rest and exercise. Fed Proc. 1986;45(13):2924-2929.

43. DiGirolamo M, Newby FD, Lovejoy J. Lactate production in adipose tissue: a regulated function with extra-adipose implications. FASEB J. 1992; 6(7):2405-2412.

44. Potthoff MJ, et al. FGF15/19 regulates hepatic glucose metabolism by inhibiting the CREB-PGC-1alpha pathway. Cell Metab. 2011;13(6):729-738.

45. Kohli R, et al. Intestinal adaptation after ileal interposition surgery increases bile acid recycling and protects against obesity-related comorbidities. Am J Physiol Gastrointest Liver Physiol. 2010; 299(3):G652-G660.

46. Patti ME, et al. Serum bile acids are higher in humans with prior gastric bypass: potential contribution to improved glucose and lipid metabolism. Obesity (Silver Spring). 2009;17(9):1671-1677.

47. Jansen PL, et al. Alterations of hormonally active fibroblast growth factors after Roux-en-Y gastric bypass surgery. Dig Dis. 2011;29(1):48-51.

48. Pournaras DJ, et al. The role of bile after Roux -en-Y gastric bypass in promoting weight loss and improving glycaemic control. Endocrinology. 2012; 153(8):3613-3619.

49. Mingrone $G$, et al. Bariatric surgery versus conventional medical therapy for type 2 diabetes. $N$ Engl J Med. 2012;366(17):1577-1585.

50. Pinto S, et al. Rapid rewiring of arcuate nucleus feeding circuits by leptin. Science. 2004; 304(5667):110-115

51 . Huszar D, et al. Targeted disruption of the melanocortin-4 receptor results in obesity in mice. Cell. 1997; 88:131-141.

52. Franklin B, Paxinos G. The Mouse Brain in Stereotaxic Coordinates. San Diego, California, USA: Academic Press; 1997.

53. Ayala JE, Bracy DP, McGuinness OP, Wasserman $\mathrm{DH}$. Considerations in the design of hyperinsulinemic-euglycemic clamps in the conscious mouse. Diabetes. 2006;55(2):390-397. 